\title{
A Novel Approach to Substituted 2H-Azirines
}

Alan R. Katritzky, * Mingyi Wang, Chavon R. Wilkerson and Hongfang Yang

Department of Chemistry, Center for Heterocyclic Compounds,

University of Florida, Gainesville, FL 32611-7200

Katritzky@chem.ufl.edu

Supporting Information 


\section{General procedure for the oximes 2 a c.}

To a solution of hydroxylamine hydrochloride $(30 \mathrm{mmol})$ in water $(50 \mathrm{~mL})$, was added a solution of benzotriazolylmethylketone $1(15 \mathrm{mmol})$ in ethanol $(50 \mathrm{~mL})$ followed by dropwise addition of $10 \% \mathrm{NaOH}(30 \mathrm{mmol})$ solution at room temperature. After addition, the yellow or off-white colored mixture was stirred under reflux overnight. Upon cooling to room temperature, white crystals of the product formed and were collected by filtration with no further purification necessary.

2-(1H-1,2,3-Benzotriazol-1-yl)-1-phenyl-1-ethanoneoxime (2a). White prisms (93\%), mp $234236{ }^{\circ} \mathrm{C}$ (lit. $\left.{ }^{1} 223225{ }^{\circ} \mathrm{C}, 56 \%\right) ;{ }^{1} \mathrm{H}$ NMR (DMSO-d $) 12.2(\mathrm{~s}, 1 \mathrm{H}), 8.00(\mathrm{~d}, J=8.4 \mathrm{~Hz}, 1 \mathrm{H}), 7.83$ $(\mathrm{d}, J=8.2 \mathrm{~Hz}, 1 \mathrm{H}), 7.737 .70(\mathrm{~m}, 2 \mathrm{H}), 7.55(\mathrm{dd}, J=7.2,7.9 \mathrm{~Hz}, 1 \mathrm{H}), 7.407 .32(\mathrm{~m}, 4 \mathrm{H}), 6.09$ (s, $2 \mathrm{H}) ;{ }^{13} \mathrm{C}$ NMR (DMSO- $\left.d_{6}\right)$ 151.2, 145.3, 134.4, 133.3, 129.6, 128.8, 127.9, 126.7, 124.5, 119.6, 110.9, 41.5.

2-(1H-1,2,3-Benzotriazol-1-yl)-1-(4-chlorophenyl)-1-ethanoneoxime (2b). White needles (91\%), mp $226227{ }^{\circ} \mathrm{C} ;{ }^{1} \mathrm{H}$ NMR (DMSO- $\left.d_{6}\right) 12.33(\mathrm{~s}, 1 \mathrm{H}), 8.01(\mathrm{~d}, J=8.2 \mathrm{~Hz}, 1 \mathrm{H}), 7.83(\mathrm{~d}, J=8.2$ $\mathrm{Hz}, 1 \mathrm{H}), 7.74(\mathrm{~d}, J=8.4 \mathrm{~Hz}, 2 \mathrm{H}), 7.58(\mathrm{dd}, J=7.8,7.0 \mathrm{~Hz}, 1 \mathrm{H}), 7.437 .37(\mathrm{~m}, 3 \mathrm{H}), 6.10(\mathrm{~s}, 2 \mathrm{H}) ;{ }^{13} \mathrm{C}$ NMR (DMSO- $\left.d_{6}\right) 149.9,144.8,134.0,132.8,128.4,128.0,127.4,124.0,119.1,110.4,41.0$. Anal. Calcd for $\mathrm{C}_{15} \mathrm{H}_{14} \mathrm{~N}_{4} \mathrm{O}$ : C, 67.65; H, 5.31; N, 21.04. Found: C, 67.43; H, 5.47; N, 21.02.

2-(1H-1,2,3-Benzotriazol-1-yl)-1-(4-methylphenyl)-1-ethanoneoxime $\quad(2 c) . \quad$ White microcrystals (92\%), mp $185188{ }^{\circ} \mathrm{C} ;{ }^{1} \mathrm{H}$ NMR (DMSO-d $) 12.11(\mathrm{~s}, 1 \mathrm{H}), 7.99(\mathrm{~d}, J=8.4 \mathrm{~Hz}, 1 \mathrm{H})$, $7.82(\mathrm{~d}, J=8.4 \mathrm{~Hz}, 1 \mathrm{H}), 7.627 .53(\mathrm{~m}, 3 \mathrm{H}), 7.37(\mathrm{dd}, J=8.0,7.3 \mathrm{~Hz}, 1 \mathrm{H}), 7.12(\mathrm{~d}, J=8.0 \mathrm{~Hz}, 2 \mathrm{H})$, $6.07(\mathrm{~s}, 2 \mathrm{H}), 2.24(\mathrm{~s}, 3 \mathrm{H}) ;{ }^{13} \mathrm{C}$ NMR $\left(\mathrm{DMSO}-d_{6}\right) 150.6,144.9,138.8,132.8,131.1,128.9,127.4,126.2$, 124.0, 119.1, 110.4, 41.0, 20.7. Anal. Calcd for $\mathrm{C}_{14} \mathrm{H}_{11} \mathrm{ClN}_{4} \mathrm{O}$ : C, 58.65; H, 3.87; N, 19.54. Found: C, 58.47; H, 3.92; N, 19.49. 
General procedure for 2-(benzotriazol-1-yl)-2H-azirines 4 a c.

To a solution of oxime $2(20 \mathrm{mmol})$ in a mixture of ethyl ether $(150 \mathrm{~mL})$ and chloroform $(50$ $\mathrm{mL})$, was added dropwise aqueous $\mathrm{KOH}\left(11.2 \mathrm{~g} \mathrm{KOH}\right.$ dissolved in $50 \mathrm{~mL}$ of water) at $0{ }^{\circ} \mathrm{C}$. After the addition of the base solution, the mixture was stirred vigorously at this temperature for $30 \mathrm{~min}$, and then a catalytic amount of $\mathrm{Bu}_{4} \mathrm{NHSO}_{4}$ was added. A solution of $p$-toluenesulfonyl chloride (TsCl) in $\mathrm{Et}_{2} \mathrm{O}(50 \mathrm{~mL})$ was added dropwise at $0{ }^{\circ} \mathrm{C}$. After this addition, the final mixture was stirred at $05{ }^{\circ} \mathrm{C}$ for $6 \mathrm{~h}$ until the solid reactants disappeared. The reaction mixture was transferred into a separatory funnel and extracted with ethyl ether. The combined organic layer was washed with water and dried over $\mathrm{MgSO}_{4}$. The residue on removal of solvents was purified by column (hexane-ethyl acetate) to give the desired products $\mathbf{4 a}$ c.

1-(3-Phenyl-2H-aziren-2-yl)-1H-1,2,3-benzotriazole (4a). White needles (40\%), mp 99100 ${ }^{\circ} \mathrm{C} ;{ }^{1} \mathrm{H}$ NMR $8.15(\mathrm{~d}, J=6.7 \mathrm{~Hz}, 2 \mathrm{H}), 8.05(\mathrm{~d}, J=8.4 \mathrm{~Hz}, 1 \mathrm{H}), 7.737 .62(\mathrm{~m}, 4 \mathrm{H}), 7.50(\mathrm{t}, J=7.6$ $\mathrm{Hz}, 1 \mathrm{H}), 7.37(\mathrm{dd}, J=7.9,7.3 \mathrm{~Hz}, 1 \mathrm{H}), 5.19(\mathrm{~s}, 1 \mathrm{H}) ;{ }^{13} \mathrm{C} \mathrm{NMR} \quad 164.6,146.1,134.4,132.8,130.5$, 129.5, 127.8, 124.2, 123.0, 120.1, 109.8, 42.7. Anal. Calcd for $\mathrm{C}_{14} \mathrm{H}_{10} \mathrm{~N}_{4}: \mathrm{C}, 71.78 ; \mathrm{H}, 4.30 ; \mathrm{N}, 23.92$. Found: C, 71.81; H, 4.39; N, 23.92.

1-[3-(4-Chlorophenyl)-2H-aziren-2-yl]-1H-1,2,3-benzotriazole (4b). Yellow prism (58\%), mp $158160{ }^{\circ} \mathrm{C} ;{ }^{1} \mathrm{H}$ NMR $8.10(\mathrm{~d}, J=8.5 \mathrm{~Hz}, 2 \mathrm{H}), 8.06(\mathrm{~d}, J=8.7 \mathrm{~Hz}, 1 \mathrm{H}), 7.75(\mathrm{~d}, J=8.4 \mathrm{~Hz}$ $1 \mathrm{H}), 7.63(\mathrm{~d}, J=8.5 \mathrm{~Hz}, 2 \mathrm{H}), 7.52(\mathrm{t}, J=7.6 \mathrm{~Hz}, 1 \mathrm{H}), 7.39(\mathrm{dd}, J=8.0,7.3 \mathrm{~Hz}, 1 \mathrm{H}), 5.17(\mathrm{~s}, 1 \mathrm{H}) ;{ }^{13} \mathrm{C}$ NMR $163.8,146.2,140.9,132.9,131.7,130.0,127.9,124.3,121.6,120.2,109.7,42.4$. Anal. Calcd for $\mathrm{C}_{14} \mathrm{H}_{9} \mathrm{ClN}_{4}$ : C, 62.58; H, 3.38; N, 20.85. Found: C, 62.81; H, 3.34; N, 20.84.

1-[3-(4-Methylphenyl)-2H-aziren-2-yl]-1H-1,2,3-benzotriazole (4c). Yellow microcrystals (66\%), mp $101102{ }^{\circ} \mathrm{C} ;{ }^{1} \mathrm{H}$ NMR $8.078 .02(\mathrm{~m}, 3 \mathrm{H}), 7.69(\mathrm{~d}, J=8.2 \mathrm{~Hz}, 1 \mathrm{H}), 7.507 .44(\mathrm{~m}, 3 \mathrm{H})$, $7.37(\mathrm{dd}, J=7.8,7.4 \mathrm{~Hz}, 1 \mathrm{H}), 5.18(\mathrm{~s}, 1 \mathrm{H}), 2.5(\mathrm{~s}, 3 \mathrm{H}) .{ }^{13} \mathrm{C} \mathrm{NMR} \quad 164.1,146.1,145.6,132.8,130.6$ 
130.2, 127.7, 124.2, 120.3, 120.1, 109.9, 42.6, 22.0. Anal. Calcd for $\mathrm{C}_{15} \mathrm{H}_{12} \mathrm{~N}_{4}: \mathrm{C}, 72.56 ; \mathrm{H}, 4.87 ; \mathrm{N}$, 22.57. Found: C, 72.63; H, 4.96; N, 24.46.

\section{General procedure for $2 H$-azirine 5 a f.}

Under $\mathrm{N}_{2}$, activated $\mathrm{Mg}$ powder (2 mmol) was added into a dried two-necked flask equipped

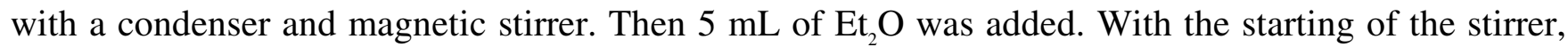
34 drops of a solution of the bromide $(2 \mathrm{mmol})$ in $5 \mathrm{~mL}$ of $\mathrm{Et}_{2} \mathrm{O}$ was added by syringe, and several minutes later, the remaining bromide solution was added dropwise. The mixture was heated and refluxed slowly for $1 \mathrm{~h}$. Upon cooling to $-18{ }^{\circ} \mathrm{C}$ (ice-salt bath), $\mathrm{ZnCl}_{2}$ solution $(1.0 \mathrm{M}, 2.0 \mathrm{~mL})$ was added, and 3040 minutes later, a solution of starting $2 H$-azirine $4(0.5 \mathrm{mmol})$ in $5 \mathrm{~mL}$ of toluene was added at this temperature. After these additions, the final mixture was stirred at $-18{ }^{\circ} \mathrm{C}$ to room temperature overnight (around $24 \mathrm{~h}$ ). TLC showed almost no starting material. The reaction was quenched with water, and the reaction mixture was diluted with $\mathrm{Et}_{2} \mathrm{O}$, washed with water, and dried over $\mathrm{MgSO}_{4}$. The residue on removal of solvents was purified by column (hexane-ethyl acetate) to give the desired products $\mathbf{5 a} \mathbf{f}$.

2-Benzyl-3-phenyl-2H-azirine (5a). Oil (70\%); ${ }^{1} \mathrm{H}$ NMR 7.777 .75 (m, 2H), 7.557 .47 (m, 3H), $7.317 .21(\mathrm{~m}, 5 \mathrm{H}), 3.06(\mathrm{dd}, J=14.6,5.1,1 \mathrm{H}), 2.76(\mathrm{dd}, J=14.6,5.1 \mathrm{~Hz}, 1 \mathrm{H}), 2.51(\mathrm{t}, J=5.1$ $\mathrm{Hz}, 1 \mathrm{H}) ;{ }^{13} \mathrm{C} \mathrm{NMR} \quad 171.6,139.3,132.8,129.3,129.0,128.9,128.5,126.3,125.5,40.1,33.2$.

2-(4-Methylbenzyl)-3-phenyl-2H-azirine (5b). Oil (70\%); ${ }^{1} \mathrm{H}$ NMR 7.867 .83 (m, 2H), $7.657 .55(\mathrm{~m}, 3 \mathrm{H}), 7.25(\mathrm{~d}, J=8.0 \mathrm{~Hz}, 2 \mathrm{H}), 7.18(\mathrm{~d}, J=8.0 \mathrm{~Hz}, 2 \mathrm{H}), 3.08(\mathrm{dd}, J=14.64 .9 \mathrm{~Hz}, 1 \mathrm{H})$, $2.77(\mathrm{dd}, J=14.6,5.3 \mathrm{~Hz}, 1 \mathrm{H}), 2.55(\mathrm{t}, J=5.1 \mathrm{~Hz}, 1 \mathrm{H}), 2.40(\mathrm{~s}, 3 \mathrm{H}) ;{ }^{13} \mathrm{C} \mathrm{NMR} \quad 171.7,136.2,135.8$, 132.8, 129.4, 129.2, 129.0, 128.8, 125.6, 39.7, 33.4, 21.0. Anal. Calcd for $\mathrm{C}_{16} \mathrm{H}_{15} \mathrm{~N}$ : C, 86.84; $\mathrm{H}, 6.83$; N, 6.33. Found: C, 86.53; H, 7.24; N, 6.20. 
2-Benzyl-3-(4-chlorophenyl)-2H-azirine (5c). Oil (70\%); ${ }^{1} \mathrm{H}$ NMR 7.67 (d, $\left.J=8.5 \mathrm{~Hz}, 2 \mathrm{H}\right)$,

$7.48(\mathrm{~d}, J=8.4 \mathrm{~Hz}, 2 \mathrm{H}), 7.337 .20(\mathrm{~m}, 5 \mathrm{H}), 3.09(\mathrm{dd}, J=14.6,4.9 \mathrm{~Hz}, 1 \mathrm{H}), 2.73(\mathrm{dd}, J=14.6,5.1$

$\mathrm{Hz}, 1 \mathrm{H}), 2.54(\mathrm{t}, J=5.1 \mathrm{~Hz}, 1 \mathrm{H}) ;{ }^{13} \mathrm{C} \mathrm{NMR} \quad 170.9,139.1,139.0,130.5,129.5,128.9,128.6,126.4$,

124.0, 40.0, 33.5. Anal. Calcd for $\mathrm{C}_{15} \mathrm{H}_{12} \mathrm{ClN}$ : C, 74.53; H, 5.00; N, 5.79. Found: C, 74.55; H, 5.37; N, 5.76.

3-(4-Chlorophenyl)-2-(4-methylbenzyl)-2H-azirine (5d). White prism (80\%), mp $9395{ }^{\circ} \mathrm{C}$;

${ }^{1} \mathrm{H}$ NMR $7.69(\mathrm{~d}, J=8.5 \mathrm{~Hz}, 2 \mathrm{H}), 7.49(\mathrm{~d}, J=8.4 \mathrm{~Hz}, 2 \mathrm{H}), 7.177 .09(\mathrm{~m}, 4 \mathrm{H}), 3.05(\mathrm{dd}, J=14.6$, $4.8 \mathrm{~Hz}, 1 \mathrm{H}), 2.68(\mathrm{dd}, J=14.6,5.4 \mathrm{~Hz}, 1 \mathrm{H}), 2.51(\mathrm{dd}, J=5.1,4.8 \mathrm{~Hz}, 1 \mathrm{H}), 2.33(\mathrm{~s}, 3 \mathrm{H}) ;{ }^{13} \mathrm{C} \mathrm{NMR}$ $171.0,139.0,136.0,135.9,130.5,129.5,129.2,128.8,124.1,39.6,33.6,21.0$. Anal. Calcd for $\mathrm{C}_{16}$ $\mathrm{H}_{14} \mathrm{ClN}: \mathrm{C}, 75.14 ; \mathrm{H}, 5.52 ; \mathrm{N}, 5.48$. Found: C, 75.18; H, 5.65; N, 5.32.

2-Benzyl-3-(4-methylphenyl)-2H-azirine (5e). Oil (71\%); ${ }^{1} \mathrm{H}$ NMR 7.57 (d, $\left.J=7.8 \mathrm{~Hz}, 2 \mathrm{H}\right)$, $7.247 .14(\mathrm{~m}, 7 \mathrm{H}), 2.97(\mathrm{dd}, J=14.6,5.1 \mathrm{~Hz}, 1 \mathrm{H}), 2.65(\mathrm{dd}, J=14.6,5.1 \mathrm{~Hz}, 1 \mathrm{H}), 2.40(\mathrm{t}, J=5.1 \mathrm{~Hz}$, 1H), 2.35 (s, 3H). ${ }^{13} \mathrm{C}$ NMR $171.1,143.6,139.5,129.7,129.4,128.9,128.5,126.2,122.7,40.2,32.9$, 21.8. Anal. Calcd for $\mathrm{C}_{16} \mathrm{H}_{15} \mathrm{~N}$ : C, 86.84; H, 6.83; N, 6.33. Found: C, 86.67; H, 7.23; N, 6.02.

2-(4-Methylbenzyl)-3-(4-methylphenyl)-2H-azirine (5f). Oil (50\%); ${ }^{1} \mathrm{H}$ NMR $7.67(\mathrm{~d}, J=$ $8.0 \mathrm{~Hz}, 2 \mathrm{H}), 7.32(\mathrm{~d}, J=8.0 \mathrm{~Hz}, 2 \mathrm{H}), 7.18(\mathrm{~d}, J=8.0 \mathrm{~Hz}, 2 \mathrm{H}), 7.12(\mathrm{~d}, J=8.0 \mathrm{~Hz}, 2 \mathrm{H}), 3.01(\mathrm{dd}, J=$ 14.6, 4.9 Hz, 1H), $2.68(\mathrm{dd}, J=14.6,5.4 \mathrm{~Hz}, 1 \mathrm{H}), 2.462 .44(\mathrm{~m}, 4 \mathrm{H}), 2.34(\mathrm{~s}, 3 \mathrm{H}) .{ }^{13} \mathrm{C} \mathrm{NMR} \quad 171.3$, 143.6, 136.4, 135.7, 129.8, 129.4, 129.2, 128.8, 122.9, 39.9, 33.1, 21.8, 21.0. HRMS (FAB) Calcd for $\mathrm{C}_{17} \mathrm{H}_{17} \mathrm{~N}_{1}\left[\mathrm{M}^{+}\right]: 235.1361$. Found: 235.1359.

\section{General procedure for $2 \mathrm{H}$-azirine $6 \mathrm{a}$ c.}

To a solution of potassium phthalimide $(0.5 \mathrm{mmol})$ in DMF $(5 \mathrm{~mL})$, was added dropwise a solution of starting $2 \mathrm{H}$-azirine $4(0.5 \mathrm{mmol})$ in DMF $(5 \mathrm{~mL})$ at room temperature. After addition, the reaction mixture was stirred at room temperature overnight (around 18h). Crushed ice was added to 
quench the reaction, then the mixture was transferred to a separatory funnel and extracted with ether. The organic layer was washed with water, dried over $\mathrm{MgSO}_{4}$. The residue on the removal of $\mathrm{Et}_{2} \mathrm{O}$ was purified by column to give yellow oil. Recrystallization was carried out in hexane/ethyl acetate.

2-(3-Phenyl-2H-aziren-2-yl)-1H-isoindole-1,3(2H)-dione (6a). Yellow microcrystals (70\%), mp $139140{ }^{\circ} \mathrm{C} ;{ }^{1} \mathrm{H}$ NMR $8.108 .07(\mathrm{~m}, 2 \mathrm{H}), 7.827 .80(\mathrm{~m}, 2 \mathrm{H}), 7.727 .70(\mathrm{~m}, 2 \mathrm{H}), 7.667 .58$ (m, 3H), 4.27 (s, 1H); ${ }^{13} \mathrm{C}$ NMR 167.9, 165.3, 134.3, 133.4, 131.7, 130.2, 128.9, 124.7, 123.3, 35.6. Anal. Calcd for $\mathrm{C}_{16} \mathrm{H}_{10} \mathrm{~N}_{2} \mathrm{O}: \mathrm{C}, 73.27 ; \mathrm{H}, 3.84 ; \mathrm{N}, 10.68$. Found: C, 73.16; H, 3.83; N, 10.58 .

2-[3-(4-Chlorophenyl)-2H-aziren-2-yl]-1H-isoindole-1,3(2H)-dione (6b). Yellow needles (57\%), mp $182.5184 .5{ }^{\circ} \mathrm{C} ;{ }^{1} \mathrm{H}$ NMR $8.03(\mathrm{~d}, J=8.4 \mathrm{~Hz}, 2 \mathrm{H}), 7.857 .81(\mathrm{~m}, 2 \mathrm{H}), 7.757 .72(\mathrm{~m}$, 2H), $7.60(\mathrm{~d}, J=8.4 \mathrm{~Hz}, 2 \mathrm{H}), 4.27(\mathrm{~s}, 1 \mathrm{H}) .{ }^{13} \mathrm{C} \mathrm{NMR} \quad 167.9,164.7,139.9,134.4,131.8,131.5,129.5$, 123.5, 123.3, 35.7. HRMS (FAB) Calcd for $\mathrm{C}_{16} \mathrm{H}_{9} \mathrm{~N}_{2} \mathrm{O}_{2}\left[\mathrm{M}^{+}\right]: 296.0353$. Found: 296.0351.

2-[3-(4-Methylphenyl)-2H-aziren-2-yl]-1H-isoindole-1,3(2H)-dione (6c). Yellow needles (79\%), mp $145146{ }^{\circ} \mathrm{C} ;{ }^{1} \mathrm{H}$ NMR 7.97 (d, J=8.1 Hz, 2H), 7.837 .79 (m, 2H), 7.727 .70 (m, 2H), $7.41(\mathrm{~d}, J=8.0 \mathrm{~Hz}, 2 \mathrm{H}), 4.24(\mathrm{~s}, 1 \mathrm{H}), 2.48$ (s. 3H). ${ }^{13} \mathrm{C} \mathrm{NMR} \quad 168.0,164.8,144.4,134.3,131.8$, 130.3, 129.7, 123.4, 122.0, 35.5, 21.9. Anal. Calcd for $\mathrm{C}_{17} \mathrm{H}_{12} \mathrm{~N}_{2} \mathrm{O}_{2}: \mathrm{C}, 73.90 ; \mathrm{H}, 4.38 ; \mathrm{N}, 10.14$. Found: C, 73.79; H, 4.21; N, 10.10.

\section{The procedure for $2 \mathrm{H}$-azirine 7 .}

To a solution of sodium salt of benzenethiol $(0.5 \mathrm{mmol})$ in $\mathrm{DMF}(5 \mathrm{~mL})$ cooled at $0{ }^{\circ} \mathrm{C}$, was added dropwise a solution of starting $2 \mathrm{H}$-azirine $\mathbf{4 a}(0.5 \mathrm{mmol})$ in DMF $(5 \mathrm{~mL})$. After addition, the reaction mixture quickly turned purple and was stirred at $0{ }^{\circ} \mathrm{C}$ for $1 \mathrm{~h}$. Crushed ice was added to quench the reaction, then the mixture was transferred to a separatory funnel and extracted with ether. The organic layer was washed with water, dried over $\mathrm{MgSO}_{4}$. The residue on the removal of $\mathrm{Et}_{2} \mathrm{O}$ was purified by column to give green oil. This compound is not stable at room temperature. 
Phenyl 3-phenyl-2H-aziren-2-ylsulfide 7. Green oil (70\%); ${ }^{1} \mathrm{H}$ NMR 7.87 (d, $\left.J=8.4 \mathrm{~Hz}, 2 \mathrm{H}\right)$, $7.677 .55(\mathrm{~m}, 5 \mathrm{H}), 7.367 .24(\mathrm{~m}, 3 \mathrm{H}), 3.84(\mathrm{~s}, 1 \mathrm{H}) ;{ }^{13} \mathrm{C}$ NMR 166.4, 136.3, 133.6, 130.1, 129.8, 129.2, 129.0, 126.8, 124.0, 37.6. HRMS (FAB) Calcd for $\mathrm{C}_{14} \mathrm{H}_{11} \mathrm{NS}[\mathrm{M}+2 \mathrm{H}]:$ 227.0769. Found: 227.0764 .

\section{References}

1. Katritzky, A. R.; Wu, J.; Wrobel, L.; Rachwal, S.; Steel, P. J. Acta Chem. Scand. 1993, 47, 167.

2. Banert, K.; Hagedorn, M.; Liedtke, C.; Melzer, A.; Schöffler, C. Eur. J. Org. Chem. $2000,257$. 